compared to a women whose main sexual partner did not travel. Having experienced genital lesions was also associated with HSV-2 infection (P-value $=0.060$ ).

Conclusion The $6.7 \%$ HSV-2 prevalence was similar to results obtained in other studies measuring the prevalence of HSV-2 among pregnant women in India. It is plausible that most women in this study contracted HSV-2 from their husbands and few women regularly use condoms with their husbands. This highlights the need for continuing education among both women and men living in rural India to increase condom use to decrease the spread of both HSV-2 and HIV.

\section{P3.041 IDENTIFYING AND CHARACTERIZING PLACES FOR THE TARGETED CONTROL OF HIV IN URBAN AREAS}

doi:10.1136/sextrans-2013-051184.0501

'S Polk, ${ }^{1,2} \mathrm{~J} \mathrm{M}$ Ellen, ${ }^{2} \mathrm{C}$ Fichtenberg, ${ }^{1} \mathrm{~S}$ Huettner, ${ }^{2} \mathrm{M}$ Reilly, ${ }^{2} \mathrm{~J}$ Parekh, ${ }^{1,2} \mathrm{~J} \mathrm{M}$ Jennings. 'Johns Hopkins School of Medicine, Baltimore, MD, United States; 'Johns Hopkins Bloomberg School of Public Health, Baltimore, MD, United States

Background HIV transmission has been associated with specific populations in specific places, i.e. places where drug and sexual networks overlap consistently. Canvassing places or venues where people congregate has been shown to be an effective means of identifying sexually transmitted infection cases for public health control and surveillance purposes. The objectives of this study were to identify and characterise heterosexual sex partner meeting venues in one U.S. urban area according to according to the presence or absence of HIV cases.

Methods Eighty-seven potential high-HIV-risk heterosexual sex partner meeting venues were identified using a three-phase methodology. Subsequently, a venue-based, cross-sectional study of sexually active 18-35 year olds was conducted in Baltimore, Maryland from October 2008 through December 2009.

Results 1,594 participants were enrolled at 87 venues. Fifty-nine HIV cases were identified yielding an overall HIV prevalence of $3.7 \%$ and a mean venue prevalence of $3.6 \%$ (range $=0 \%$ to $25 \%$ ). One or more cases of HIV was identified at $42 \%(n=37)$ of venues (i.e. HIV positive venues). In bivariate analysis, commercial sex work (OR: 1.04; 95\% CI: 1.01, 1.08), high HIV-risk sexual partnering (OR: 1.11 ; 95\% CI: 1.03, 1.20), recent parenteral risk behaviour (OR: 1.05; 95\% CI: 1.01, 1.10) and drug market activity (OR: 2.67; $95 \%$ CI: $1.09,6.52)$ were reported significantly more frequently at HIV positive versus HIV negative venues. In final age-adjusted models, HIV positive venues had $10 \%$ more high HIV-risk sexual partnering (OR: 1:10; 95\% CI: 1.01, 1.19) and more than twice as much drug market activity (OR: 2.59; 95\% CI: 1.04, 6.46) compared to HIV negative venues. Sex market activity was not significantly associated with venue HIV status.

Conclusions This study highlights characteristics of venues, such as drug markets, that may be important in identifying places which are more likely to have active HIV transmission.

\section{P3.042 HIV PREVENTION IN MOTHERS AND CHILDREN OF DELHI SLUMS: LEARNING, PERSPECTIVES AND LOOKING AHEAD}

doi:10.1136/sextrans-2013-051184.0502

\section{A Bajaj. Cygnus Medicare, New Delhi, India}

The most effective way to prevent mother-to-child transmission of HIV involves antiretroviral drugs and avoidance of breastfeeding, When women who are already on ART become pregnant, the benefits and risks of ART in the first trimester need to be considered. The benefits are a reduction in the risk of developing resistance and a decrease in the risk to the mother. The risk consists of the potential for ARV fetal toxicity. Good clinical management of HIV in pregnant women requires the support of a multidisciplinary team, including antenatal specialists, paediatricians, counsellors and community-based organisations.

Cygnus Medicare, a newly emerging healthcare organisation dedicated to providing world class healthcare in resource-limited settings, has been working in the slums of Delhi for last several years with a strong public health approach. It runs seven fully equipped hospitals where poor people are treated at subsidised rates and special programmes are held which focus on education of high risk groups, including commercial sex workers and migrant workers on HIV. Daily ante-natal clinics are held, benefitting over 5000 women in last 3 years. Ante natal clinics and delivering rooms have a dedicated team of health workers trained in education on PMTCT. A large number of awareness camps and activities are organised. The entire medical team periodically goes through trainings and orientations focusing on education and prevention of HIV/AIDS with an emphasis on PMTCT.

The organisation also has an outreach component of service delivery, through which remote slum areas are regularly visited by mobile medical vans to provide curative, diagnostic and educational services to the pregnant women.

The response and acceptance to the programme so far has been very positive. Over the last three years, there has been a large increase in the number of women attending the ante-natal clinics and camps.

\section{P3.043 PRETERM BIRTH AND FETAL GROWTH RESTRICTION IN HIV-INFECTED PREGNANT WOMEN AT A TERTIARY PUBLIC HOSPITAL IN VITÓRIA, BRAZIL}

doi:10.1136/sextrans-2013-051184.0503

1,2H B dos Reis, 'K S Araujo, 'L Ribeiro, 'D R Rocha, 'D P Rosato, ${ }^{2 M}$ R L Passos, 'P R Merçon de Vargas. ${ }^{1}$ Federal University of Espírito Santo, Vitoria, Brazil; ${ }^{2}$ Fluminense Federal University, Niteroi, Brazil

Introduction A maternal HIV infection may have two outstanding consequences to fetal health: mother-to-child transmission and adverse perinatal outcome. After the success in reducing mother-tochild transmission the attention must now be diverted to the high proportion of HIV-exposed children that are born preterm or with fetal growth restriction.

Objective To determine the prevalence of preterm birth and fetal growth restriction in low income, antiretroviral users, HIV-infected women and to verify its relation to the HIV infection stage and comorbidity.

Patients and Methods Out of 250 deliveries from HIV-infected mothers at a tertiary public university hospital in the city of Vitória, state of Espírito Santo, Southeastern Brazil, from November 2001 to May 2012, we included 109 (43.6\%) single pregnancies with data about the gestational age, HIV status, antiretroviral use, and fetal dimensions. Data were extracted from clinical and pathological records, the gestation age estimated and the fetal dimensions classified as small, adequate, and large for gestational age according to the pertinent reference standard.

Results Preterm birth was observed in $17.4 \%$, low birth weight in $23.9 \%$ and small for gestational weight, length, and cephalic and abdominal circumferences in $22.0 \%, 29.0 \%, 1.0 \%$, and $43.6 \%$, respectively, without significant variation according to the HIV infection severity or the existence of comorbidity. The concomitant distribution of small for gestational weight, length and abdominal circumference points to an asymmetrical fetal growth restriction.

Conclusions The prevalence of preterm birth and growth restriction were higher than the Espirito Santo State liveborn Registry prevalence and the expected population distribution in the gestations of HIV-infected, low income, ART users, and publicly assisted pregnant women in this casuistry. 